\title{
A Behçet's Syndrome Case with an Unusual Vascular Involvement
}

\author{
Neşe Dursunoğlu ${ }^{1}$, Ali İhsan Yıldız ${ }^{1}$, Ali Koçyiğit ${ }^{2}$, Bedriye Atay Yayla ${ }^{1}$, \\ Zahide Alaçam ${ }^{1}$, Zümrüt Çeven², Belgin Erkan Aydoğan ${ }^{1}$
}

${ }^{1}$ Department of Chest Diseases, Pamukkale University Faculty of Medicine, Denizli, Turkey

${ }^{2}$ Department of Radiology, Pamukkale University Faculty of Medicine, Denizli, Turkey

\begin{abstract}
"Behçet's Syndrome" was described as the triple-symptom complex of oral aphthous ulcers, genital ulcers and uveitis by Hulusi Behçet, a Turkish dermatologist, in 1937. It can present with vascular, cutaneous, pulmonary, neurological, rheumatologic, gastrointestinal and genitourinary system involvements. The main components of pulmonary involvement in Behçet's Syndrome are pulmonary artery aneurysms, arterial and venous thrombosis, pulmonary infarction, recurrent pneumonia, bronchitis obliterans organising pneumonia and pleurisy. This study presented a case of a young man with hemoptysis resulting from anastomosis between the bronchial arteries and pulmonary artery and parenchymal destruction, and thus had a different vascular involvement for Behçet's syndrome.
\end{abstract}

Keywords: Anastomosis, Behçet's syndrome, hemoptysis, pulmonary artery aneurysm

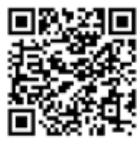

Received Date: 14.02 .2011

Accepted Date: 19.02.2012

\section{Address for correspondence}

Neşe Dursunoğlu, Department of Chest Diseases, Pamukkale University Faculty of Medicine, Denizli, Turkey

E-mail: ndursunoglu@yahoo.com

(C) Copyright 2014 Turkish Respiratory Society (TRS) Eurasian J Pulmonol 2014

DOI: 10.5152/ejp.2014.23590

- Available online at www.eurasianjpulmonol.com

\section{INTRODUCTION}

Behçet's Syndrome is a systemic disease which was described as the triple-symptom complex of oral aphthous ulcers, genital ulcers and uveitis by Turkish dermatologist Hulusi Behçet in 1937 and is characterised by vascular, cutaneous, pulmonary, neurological, rheumatologic, gastrointestinal and genitourinary system involvement. In Behçet's syndrome, pulmonary artery aneurysms, arterial and venous thrombosis, pulmonary infarction, recurrent pneumonia, bronchitis obliterans organising pneumonia and pleurisy are the main characteristics of pulmonary involvement (1). Pulmonary artery aneurysm is a rare condition, but is critically important due to the high risk of rupture and the fact that it can be life threatening. However, it is also possible to completely cure aneurysms by medical treatment (2). This case study describes a young man who had hemoptysis resulting from anastomosis between the left bronchial artery and pulmonary artery, and from parenchymal destruction, who was also diagnosed with Behçet's Syndrome with a rare vascular involvement.

\section{CASE REPORT}

A thirty-year-old male patient presented with little complaint of hemoptysis for the first time 7 months ago and had received antimicrobial therapy several times. The patient had intermittent hemoptysis, and was admitted to our clinic with a complaint of bloody sputum of approximately $300 \mathrm{ml}$, fever, shortness of breath, and weight loss. In the previous thorax computed tomography (CT) examination of the case, the appearance of a cavitary lesion in the neighbourhood of the right hilus and minimal enlargement of the pulmonary artery were detected (Figure 1). After admission to our clinic, the patient was hospitalised with the pre-diagnoses of pneumonia and tuberculosis. According to the physical examination results, his vital and respiratory system findings were normal, but an ulcerative lesion and scars were found in the scrotum. No findings were obtained in the examinations of other systems. Laboratory results of the patient were as follows: sedimentation: $62 \mathrm{~mm} /$ hour, lactate dehydrogenase (LDH): $240 \mathrm{~L} / \mathrm{L}$, antinuclear antibody (ANA): 1/100+, Anti ds-DNA: (-), PR2 and myeloperoxidase (MPO) anti-neutrophilic cytoplasmic antibody (ANCA)(-). His detailed medical history revealed that he had experienced recurrent oral aphthae for years. However, he did not have a history of uveitis. The patient with the Purified 


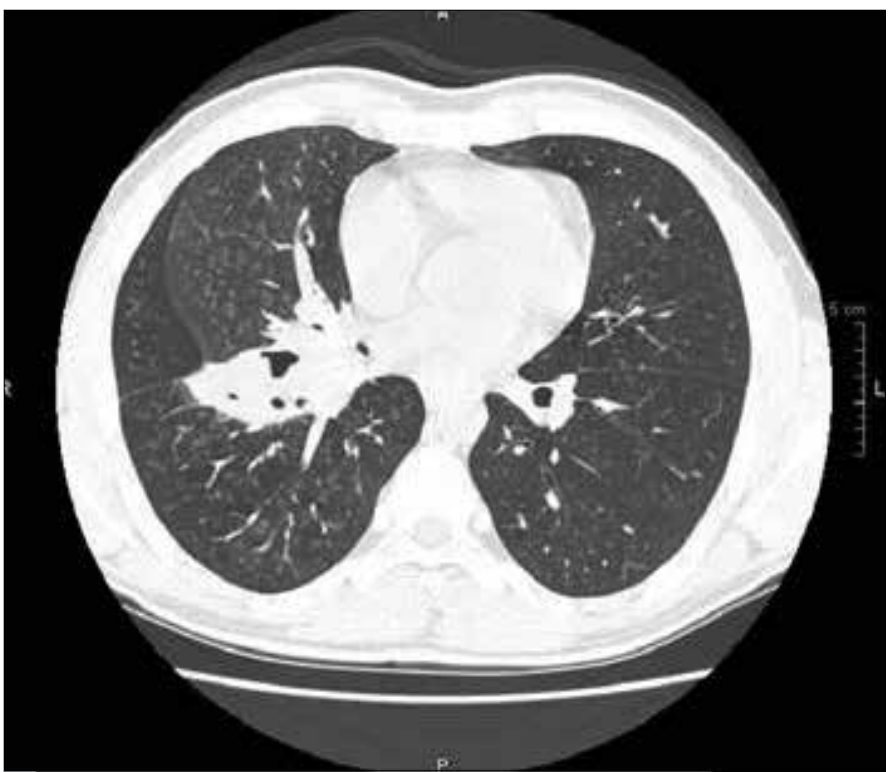

Figure 1. Thorax CT

Protein Derivative (PPD) test result of $16 \mathrm{~mm}$ was initiated on anti-tuberculosis treatment, including empirically four basic drugs because his clinical and CT findings were also consistent with pulmonary tuberculosis (PT). The patient, with the tree-in-bud appearance in the right lower lobe on thorax $\mathrm{CT}$, was exposed to bronchoscopy (Figure 2). No bleeding focus was identified with bronchoscopic examination. Transbronchial lung biopsy from the right lower lobe was performed and lavage samples were sent for TB culture. The patient, whose hemoptysis was still lasting, underwent bronchial artery angiography. In his angiography, total obliteration developed due to vasospasm which resulted from irritation in the right bronchial artery during catheterisation (Figure 3). Therefore, the embolisation that had been planned could not be performed. A week later, the second angiography was performed for the patient whose massive hemoptysis recurred and arterio-arterial anastomoses were detected between end branches of the left bronchial artery and the branches of the pulmonary artery (Figure 4). In order to control active hemoptysis, bronchial artery embolisation was carried out using 400-600 micron polyvinyl alcohol particles. Due to the fact that effective embolisation could not be provided in control angiogram, a complete luminal embolisation was provided with free coils with a size of $2 \times 10 \mathrm{~mm}$ and $4 \times 10 \mathrm{~mm}$ (Figure 5). Since the embolisation process was performed successfully and the patient's hemoptysis did not recur, pulmonary artery angiography was not needed in addition to bronchial artery angiography. In the follow-up period, the patient whose complaint of hemoptysis disappeared was initiated on therapies with cyclophosphamide and methylprednisolone for Behçet's syndrome. Hemoptysis did not recur in the controls at the outpatient clinic. Anti-TB treatment was ceased after two months due to the absence of growth in the cultures of pulmonary tuberculosis and immunosuppressive therapy was initiated.

\section{DISCUSSION}

The least common findings of Behçet's Syndrome are pulmonary findings, with a rate of $1-8 \%$ (1). The frequency rate of pulmonary artery aneurysms is $1.1 \%$ (3). They appear approximately 3.6 years after the extrapulmonary findings. Among the pulmonary findings, hemoptysis (with the frequency of $77 \%$ ) is mostly seen in young male patients and can cause over $500 \mathrm{ml}$ of bleeding $(1,4)$. The most

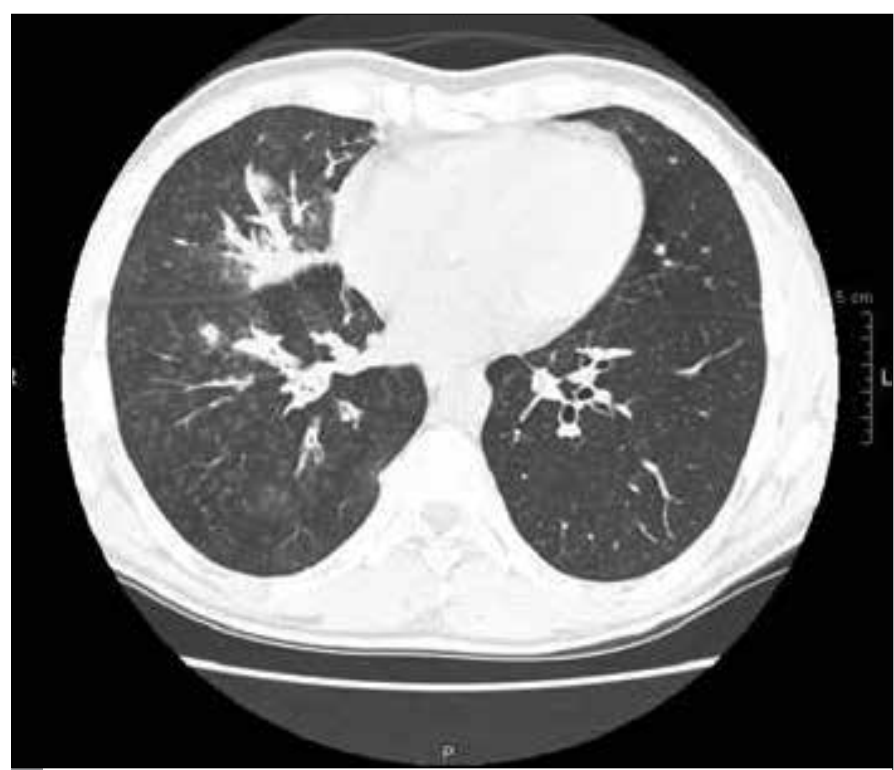

Figure 2. Tree-in-bud appearance in parenchyma on thorax CT

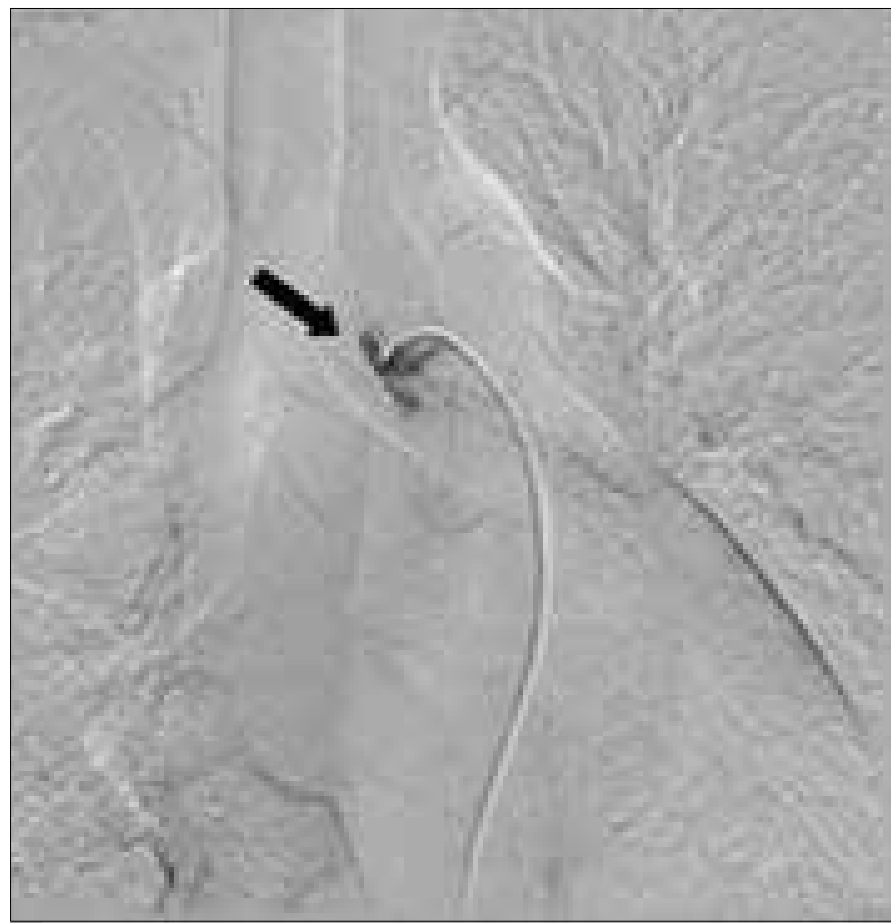

Figure 3. Bronchial artery angiography; obliterated right bronchial artery

common cause of mortality is massive hemoptysis due to pulmonary artery aneurysm (1). In the retrospective analysis of 2319 cases with Behçet's Syndrome by Küçükoğlu et al. (5), the pulmonary artery was the most frequently involved artery and its rate of involvement was found to be $3.9 \%$. Uzun et al. (6) divided pulmonary artery involvement into two groups in their study: macroscopic and microscopic involvement. They reported that macroscopic involvement included conditions involving the pulmonary artery, with life expectancy for these patients being lower than for those with microscopic involvement. Also, massive hemoptysis was not observed in any case with microscopic vascular involvement. 


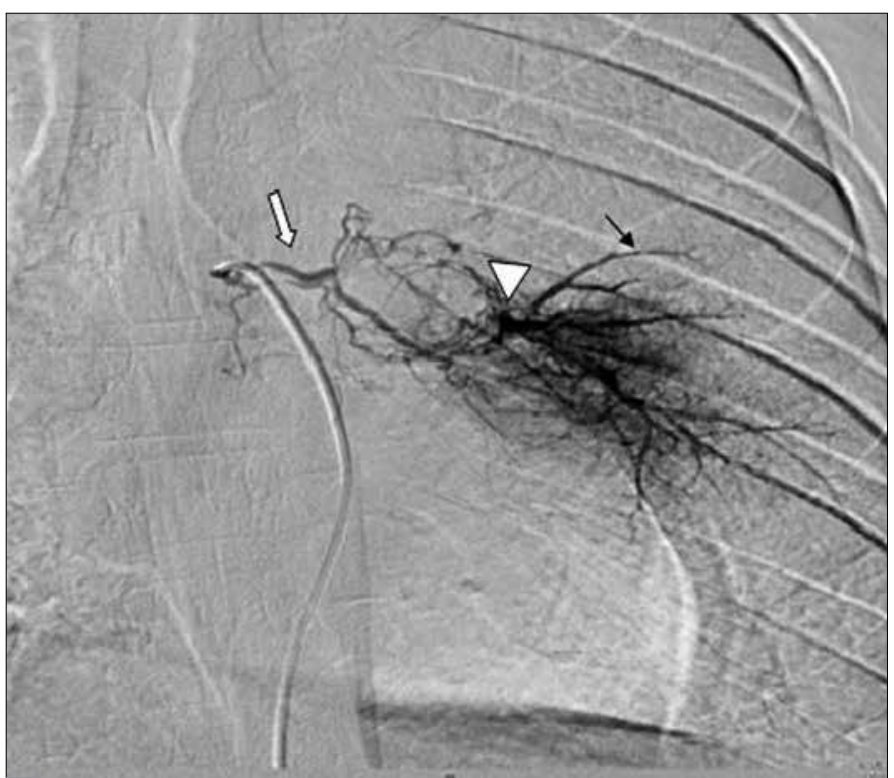

Figure 4. Bronchial artery angiography

White arrow: Left bronchial artery; White triangle: bronchial artery and pulmonary artery anastomosis; Black arrow: end branch of pulmonary artery

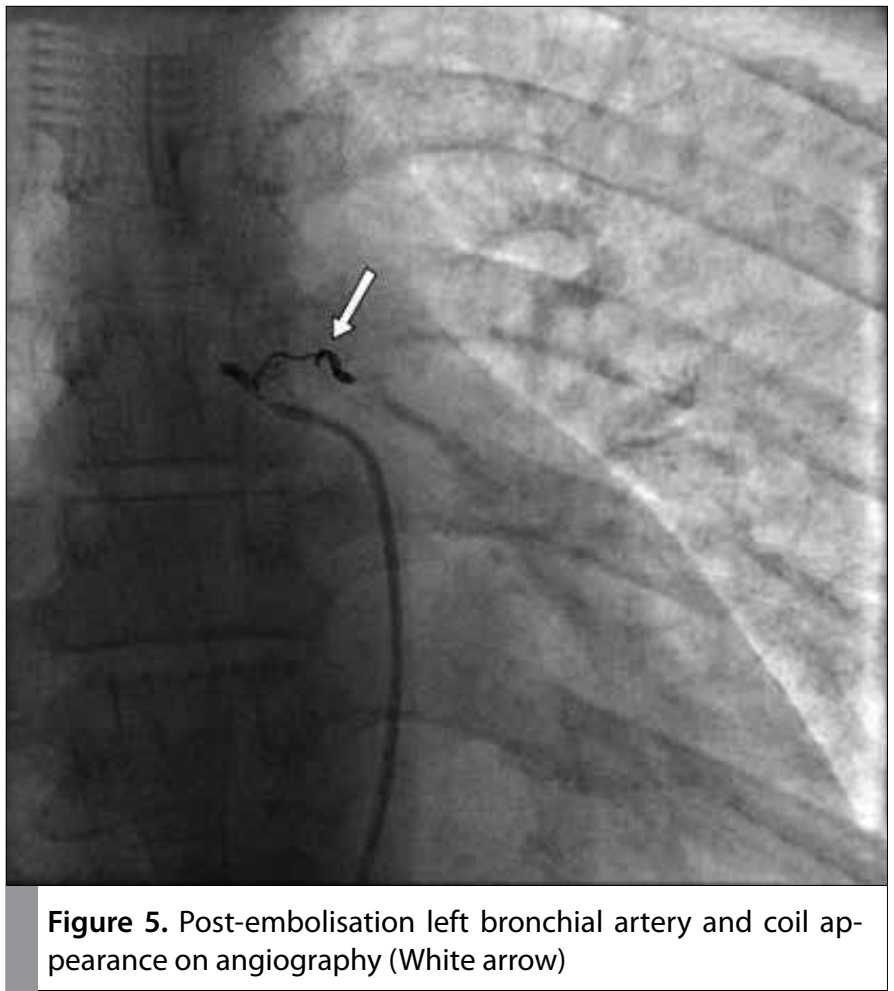

The medical history of our patient included oral aphthae, eye lesion and aphthae in the scrotum; his complaint of hemoptysis first appeared approximately 7 months ago. He had hemoptysis of $300 \mathrm{cc}$ at most. In the literature, the most frequent cause of hemoptysis is stated to be pulmonary artery aneurysm. However, in this case, the cause of hemoptysis was considered to be associated with anastomosis between the left bronchial arteries and pulmonary artery, a different vascular involvement in Behçet's Syndrome that had not been reported in literature previously, and parenchymal destruction. Pulmonary artery aneurysm was not detected on CT of the thorax; that is, macroscopic vascular involvement was not observed. In this situation, the cause of massive bleeding was thought to be the aneurysm of high-pressure blood in the bronchial artery to the pulmonary artery and parenchymal destruction. As a treatment, the bronchial artery was embolised with a coil.

Atelectasia, volume depletion, wedge-shaped or linear areas, and nodular or reticular opacities which develop in Behçet's Syndrome are thought to be associated with pulmonary hemorrhage and infarction (4). In our case, tree-in-bud appearance in the parenchyma caused us to suspect tuberculosis at the beginning. However, the finding of normal lung parenchyma on transbronchial lung biopsy, the absence of growth in the cultures of pulmonary tuberculosis, and the absence of active bleeding focus on bronchoscopy suggested that tree-in-bud appearance resulted from the aspiration of blood occurring due to parenchymal destruction associated with Behçet's Syndrome (Figure 2).

Empirical tuberculosis treatment was initiated before obtaining the culture results because the PPD value of the patient was $16 \mathrm{~mm}$. Anti-TB treatment was ceased after two months due to negative results for the pulmonary tuberculosis culture samples and the dose of immunosuppressive therapy was decreased.

\section{CONCLUSION}

Despite the fact that pulmonary artery aneurysm is the most common cause of hemoptysis in Behçet's Syndrome, bronchial artery-pulmonary artery anastomosis should be taken into consideration in those cases in which pulmonary artery aneurysm is not detected on CT. It is emphasised that embolisation accompanied by angiography in both diagnosis and treatment periods are useful for hemoptysis treatment.

\section{Ethics Committee Approval: N/A.}

Informed Consent: Written informed consent was obtained from the patient who participated in this study.

Peer-review: Externally peer-reviewed.

Author Contributions: Concept - N.D., A.I.Y.; Design - N.D., A.I.Y., A.K.; Supervision - N.D., A.K.; Resource - A.K., Z.Ç.; Materials - A.I.Y., Z.Ç.; Data Collection\&/ or Processing - A.I.Y., B.A.Y., Z.A., B.E.A.; Analysis\&/or Interpretation - N.D., A.I.Y., A.K.; Literature Search - A.I.Y., B.A.Y., Z.A., B.E.A.; Writing - N.D., A.I.Y., A.K.; Critical Reviews - N.D., A.K.

Conflict of Interest: No conflict of interest was declared by the authors.

Financial Disclosure: The authors declared that this study has received no financial support.

\section{REFERENCES}

1. Erkan F, Gul A, Tasali E. Pulmonary manifestations in Behçet's disease. Thorax 2001; 56: 572-8.

2. Tunaci M, Ozkorkmaz B, Tunaci A, Gul A, Engin G, Acunas B. CT fidings of pulmonary artery aneurysms during treatment for Behçet's disease. AJR Am J Roentgenol 1999; 172: 729-33.

3. Trombati N, Souabny A, Aichane A, Bahlaoui A, Afif H, Bouayad Z. Pulmonary arterial aneurysms revealing Behçet's disease: from diagnosis to treatment. Rev Med Interne 2002; 23: 334-41.

4. El Houari T1, Oukerraj L, Ghzaiel L, Fellat I, Azeroual M, Serraj K, et al. Management of Behçet disease with multiple complications. Hellenic J Cardiol 2009; 50: 420-22.

5. Sarica-Kucukoglu R, Akdag-Kose A, Kayaball M, Yazganoglu KD, Disci R, Erzengin $D$, et al. Vascular involvement in Behçet's disease: a retrospective analysis of 2319 cases. Int J Dermatol 2006; 45: 919-21.

6. Uzun O, Erkan L, Akpolat I, Findik S, Atici AG, Akpolat T. Pulmonary Involvement in Behçet's Disease. Respiration 2008; 75: 310-21. 